\title{
Another piece of the puzzle
}

A study of $\Lambda_{b}$ baryon decays has provided the first direct experimental evidence that spinning matter and antimatter differ. This result may help us understand the puzzling matter-antimatter imbalance in the Universe.

\section{Gauthier Durieux and Yuval Grossman}

W hat is the fundamental difference between matter and antimatter? This is the key question that physicists try to answer by studying charge-parity symmetry (CP), which relates the two. So far, evidence for $\mathrm{CP}$ violation has only been found in decays of spinless $K$ and $B$ mesons. Writing in Nature Physics, the LHCb collaboration ${ }^{1}$ reports the first evidence for $\mathrm{CP}$ violation in the decay of a particle carrying spin, the $\Lambda_{b}$ baryon (Fig. 1). This result is not exactly unexpected given the magnitude of CP violation in $B$ meson decays. But, while its quantitative compatibility with our current understanding remains to be established, it opens a whole new avenue for the investigation of $\mathrm{CP}$ violation.

At the microscopic level, the origin of the differing behaviour between matter and antimatter is very subtle. It arises from the phases in the Lagrangian and can thus only be observed in interference effects. Measuring CP violation is therefore challenging. It was first observed experimentally in 1964, in the decay of a long-lived neutral kaon, $K_{\mathrm{L}}$, into two pions ${ }^{2}$. Since then, a few more CP-violating processes have been spotted: first in additional kaon decays and, more recently, in $B$ meson decays ${ }^{3,4}$. Until now, no evidence for CP violation had been found in the decay of any other particle. However, our understanding of CP violation critically relies on the diversity of its observations. Kaon physics established the phenomenon, but a deeper understanding was only gained through its observation in $B$ meson decays. We now know that the source of the observed CP violation in both systems is the one phase of the quark mixing matrix, known as the Kobayashi-Maskawa phase ${ }^{5}$.

Although antimatter is, to a very good approximation, just a mirror image of matter, the amount of antimatter observed in the Universe is negligible compared to that of matter. CP violation is a mandatory ingredient for the generation of this asymmetry in the early Universe ${ }^{6}$. The Kobayashi-Maskawa phase would,

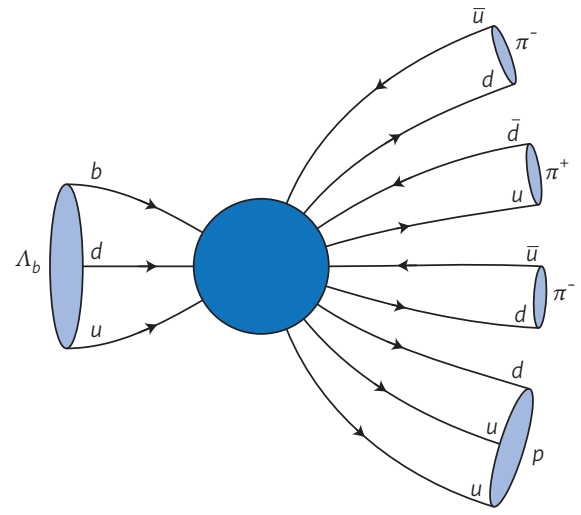

Figure 1 | The LHCb collaboration found evidence for CP violation in the decay mode $\Lambda_{b} \rightarrow p \pi^{-} \pi^{+} \pi^{-}$. The $\Lambda_{b}$ baryon is composed of a $u d b$ triad of quarks, the proton $p$ (also a baryon) is made of und quarks, and the charged pions $\pi^{ \pm}$(which are mesons) respectively contain an $u \bar{d}$ and $\bar{u} d$ quark-antiquark pair. Many different processes, represented collectively by the central blob, can contribute to this mode and reveal CP-violating phases through their interferences.

however, lead to an asymmetry at least ten orders of magnitude smaller than the one observed. This discrepancy points at the presence of additional sources of CP violation, which remain to be discovered. These may arise in the quark sector, much like the already-known phase, or come from a different sector of the theory. By examining the difference between matter and antimatter in new systems, such as baryons, one hopes to shed light on these additional sources of CP violation.

\section{The $\mathrm{LHCb}$ result is the first evidence for $\mathrm{CP}$ violation in a spin-carrying particle decay.}

The $\mathrm{LHCb}$ result provides the first evidence for CP violation in a spincarrying particle decay. It is also the second observation of $\mathrm{CP}$ violation in a 'motion-reversal-odd' observable ${ }^{7,8}$, which measures differences between the angular distributions of the particle and antiparticle decay products, instead of just comparing their total decay rates. (The first observation of this type was obtained in the decay of the $K_{\mathrm{L}}$ kaon to two pions, an electron and a positron ${ }^{9}$.) Motion-reversal-odd observables constitute a complementary means of investigation because they have different sensitivity to CP-conserving phases $^{10}$. Moreover, they do not require equally sized samples of particles and antiparticles, which is especially convenient when production rates are asymmetric like in the LHCb experiment.

The statistical significance of the $\mathrm{LHCb}$ measurement corresponds to 3.3 Gaussian standard deviations $(\sigma)$ whereas $5 \sigma$ is the self-imposed criterion for claiming a discovery. This higher significance could be reached by analysing the data already collected during the second run of the LHC. The study of other baryon decays with this larger data set could also provide insight into the sources of $\mathrm{CP}$ violation. The pioneering result reported by the $\mathrm{LHCb}$ collaboration constitutes a first step into unexplored territory. Hopefully, clues to solving the matter-antimatter conundrum lie ahead.

Gauthier Durieux is at Deutsches Elektronen Synchrotron (DESY), Notkestraße 85, D-22607 Hamburg, Germany, and Yuval Grossman is at the Laboratory for Elementary Particle Physics, Cornell University, Ithaca, New York 14853, USA. e-mail: gauthier.durieux@desy.de; yg73@cornell.edu

References

1. Aaij, R. et al. (LHCb collaboration) Nat. Phys. 13, 391-396 (2017).

2. Christenson, J. H., Cronin, J. W., Fitch, V. L. \& Turlay, R. Phys. Rev. Lett. 13, 138-140 (1964).

3. Aubert, B. et al. (BABAR collaboration) Phys. Rev. Lett. 87, 091801 (2001).

4. Abe, K. et al. (Belle collaboration) Phys. Rev. Lett. 87, 091802 (2001).

5. Kobayashi, M. \& Maskawa, T. Prog. Theor. Phys. 49, 652-657 (1973).

6. Sakharov, A. D. Pisma Zh. Eksp. Teor. Fiz. 5, 32-35 (1967).

7. Sachs, R. G. The Physics of Time Reversal (Univ. of Chicago Press, 1987).

8. Branco, G. C., Lavoura, L. \& Silva, J. P. CP Violation (Clarendon, 1999).

9. Alavi-Harati, A. et al. (KTeV collaboration) Phys. Rev. Lett. 84, 408-411 (2000).

10. Gasiorowicz, S. Elementary Particle Physics (Wiley, 1966). 